\title{
Rhodium-Catalyzed [2+2+2] Cycloaddition of 1,6-Diynes with Isothiocyanates and Carbon Disulfide
}

\author{
Ken Tanaka,* Azusa Wada, and Keiichi Noguchi
}

Department of Applied Chemistry, Graduate School of Engineering, and Instrumentation Analysis Center, Tokyo University of Agriculture and Technology, Koganei, Tokyo 184-8588, Japan

\section{General}

Anhydrous $\mathrm{CH}_{2} \mathrm{Cl}_{2}$ (No. 27,099-7) and $\left(\mathrm{CH}_{2} \mathrm{Cl}\right)_{2}$ (No. 28,450-5) were obtained from Aldrich and used as received. All reagents were obtained from commercial sources and used as received. All reactions were carried out under an atmosphere of argon or nitrogen in ovendried glassware with magnetic stirring. Diynes $\mathbf{1 a},{ }^{1} \mathbf{1} \mathbf{b},{ }^{2} \mathbf{1 c},{ }^{3}$ and $\mathbf{1} \mathbf{d}^{4}$ were prepared according to the literatures.

\section{Rhodium-Catalyzed [2+2+2] Cycloaddition of 1,6-Diynes with Isothiocyanates and Carbon Disulfide (Table 2)}

General Procedure (Table 2, entry 1). Under an Ar atmosphere, BINAP (9.3 mg, 0.015 mmol) and $[\mathrm{Rh}(\operatorname{cod}) \mathrm{Cl}]_{2}(3.7 \mathrm{mg}, 0.0075 \mathrm{mmol})$ were dissolved in $\mathrm{CH}_{2} \mathrm{Cl}_{2}(1.0 \mathrm{~mL})$ and the mixture was stirred for $5 \mathrm{~min} . \mathrm{H}_{2}$ was introduced to the resulting solution in a Schlenk tube. After stirring for $0.5 \mathrm{~h}$ at room temperature, the resulting solution was concentrated to dryness and dissolved in $\left(\mathrm{CH}_{2} \mathrm{Cl}\right)_{2}(0.5 \mathrm{~mL})$. To this solution was added dropwise over $1 \mathrm{~min}$ a solution of 2,2-dibut-2-ynylmalonic acid dimethyl ester (1a, $62.5 \mathrm{mg}, 0.30 \mathrm{mmol}$ ) and phenyl isothiocyanate $(2 \mathbf{a}, 44.6 \mathrm{mg}, 0.33 \mathrm{mmol})$ in $\left(\mathrm{CH}_{2} \mathrm{Cl}\right)_{2}(0.5 \mathrm{~mL})$ and washed remaining substrates away by using $\mathrm{CH}_{2} \mathrm{Cl}_{2}(0.5 \mathrm{~mL})$. The mixture was stirred at $80{ }^{\circ} \mathrm{C}$ for $15 \mathrm{~h}$. The resulting solution was concentrated and purified by silica gel column chromatography (hexane:EtOAc = 5:1), which furnished 3-phenylimino-3,5-dihydro-7Hcyclopentan[c]thiopyran-6,6-dicarboxylic acid dimethyl ester (3aa, $91.0 \mathrm{mg}, 0.265 \mathrm{mmol}$, $88 \%$ yield) as a yellow solid.

\section{3-Phenylimino-3,5-dihydro-7H-cyclopentan[c]thiopyran-6,6-dicarboxylic acid} dimethyl ester (3aa, entry 1, reaction time: 12 h). ${ }^{1}$ 


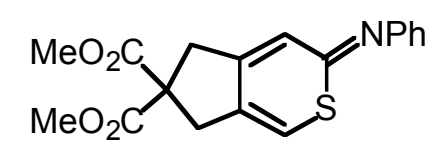

Mp 95.0-95.5 ${ }^{\circ} \mathrm{C} ;{ }^{1} \mathrm{H}$ NMR $\left(\mathrm{CDCl}_{3}, 300 \mathrm{MHz}\right) \delta 7.30-7.42(\mathrm{~m}, 2 \mathrm{H}), 7.05-7.13(\mathrm{~m}, 1 \mathrm{H})$, 6.85-6.90 (m, 2H), $6.61(\mathrm{~s}, 1 \mathrm{H}), 6.56(\mathrm{~s}, 1 \mathrm{H}), 3.78(\mathrm{~s}, 6 \mathrm{H}), 3.35(\mathrm{~d}, J=1.8 \mathrm{~Hz}, 2 \mathrm{H}), 3.24(\mathrm{~d}, J$ $=1.8 \mathrm{~Hz}, 2 \mathrm{H}) ;{ }^{13} \mathrm{C} \mathrm{NMR}\left(\mathrm{CDCl}_{3}, 75 \mathrm{MHz}\right) \delta 171.1,157.9,151.2,149.5,130.8,130.0,124.3$, $121.5,121.1,119.8,58.8,55.7,40.9,39.8$.

3-o-Tolylimino-3,5-dihydro-7H-cyclopenta[c]thiopyran-6,6-dicarboxylic acid dimethyl ester (3ab, entry 2, reaction time: $13 \mathrm{~h}$ ).

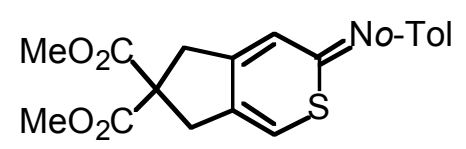

Yellow oil; IR (neat) 3300, 2900, 1710, 1530, 1420, 1235, 1200, 890, $710 \mathrm{~cm}^{-1} ;{ }^{1} \mathrm{H}$ NMR $\left(\mathrm{CDCl}_{3}, 300 \mathrm{MHz}\right) \delta 7.15-7.24(\mathrm{~m}, 2 \mathrm{H}), 7.02(\mathrm{dt}, J=7.5,1.5 \mathrm{~Hz}, 1 \mathrm{H}), 6.73(\mathrm{dd}, J=7.5,1.5$ $\mathrm{Hz}, 1 \mathrm{H}), 6.55-6.65(\mathrm{~m}, 2 \mathrm{H}), 3.76(\mathrm{~s}, 6 \mathrm{H}), 3.36(\mathrm{~d}, J=1.5 \mathrm{~Hz}, 2 \mathrm{H}), 3.23(\mathrm{~d}, J=1.5 \mathrm{~Hz}, 2 \mathrm{H})$, $2.08(\mathrm{~s}, 3 \mathrm{H}) ;{ }^{13} \mathrm{C} \mathrm{NMR}\left(\mathrm{CDCl}_{3}, 75 \mathrm{MHz}\right) \delta 170.8,157.3,149.7,149.0,131.1,130.5,128.0$, $127.2,124.0,121.3,120.8,118.7,58.7,53.1,40.8,39.7,17.2$; HRMS (EI) calcd for $\mathrm{C}_{23} \mathrm{H}_{21} \mathrm{NO}_{4} \mathrm{~S}[\mathrm{M}]^{+} 357.1035$, found 357.1025 .

3-(4-Methoxyphenylimino)-3,5-dihydro-7H-cyclopenta[c]thiopyran-6,6-dicarboxylic acid dimethyl ester (3ac, entry 3 , reaction time: $13 \mathrm{~h}$ ).

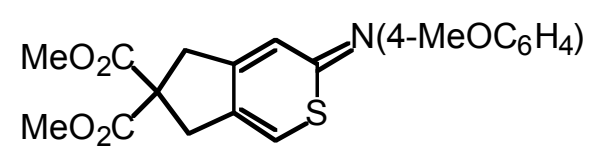

Yellow solid; Mp 153.5-154.0 ${ }^{\circ} \mathrm{C}$; IR (neat) 3000, 2850, 1710, 1530, 1480, 1200, 810 $\mathrm{cm}^{-1} ;{ }^{1} \mathrm{H}$ NMR $\left(\mathrm{CDCl}_{3}, 300 \mathrm{MHz}\right) \delta 6.88-6.97(\mathrm{~m}, 2 \mathrm{H}), 6.82-6.88(\mathrm{~m}, 2 \mathrm{H}), 6.62(\mathrm{~s}, 1 \mathrm{H}), 6.54$ $(\mathrm{s}, 1 \mathrm{H}), 3.79(\mathrm{~s}, 3 \mathrm{H}), 3.77(\mathrm{~s}, 6 \mathrm{H}), 3.34(\mathrm{~d}, J=1.8 \mathrm{~Hz}, 2 \mathrm{H}), 3.23(\mathrm{~d}, J=1.8 \mathrm{~Hz}, 2 \mathrm{H}) ;{ }^{13} \mathrm{C} \mathrm{NMR}$ $\left(\mathrm{CDCl}_{3}, 75 \mathrm{MHz}\right) \delta 170.8,157.6,156.3,148.9,144.0,130.6,121.4,120.8,120.7,114.9,58.7$, 55.3, 53.1, 40.8, 39.7; HRMS (EI) calcd for $\mathrm{C}_{19} \mathrm{H}_{19} \mathrm{NO}_{5} \mathrm{~S}[\mathrm{M}]^{+} 373.0984$, found 373.1013.

3-(4-Methoxyphenylimino)-3,5-dihydro-7H-cyclopenta[c]thiopyran-6,6-dicarboxylic acid dimethyl ester (3ad, entry 4, reaction time: 12 h). 


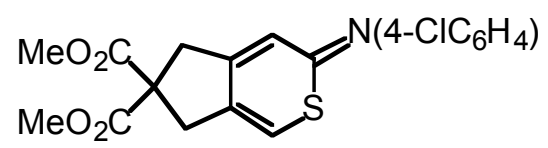

Yellow solid; Mp 153.5-154.0 ${ }^{\circ} \mathrm{C}$; IR (neat) 3000, 2850, 1710, 1530, 1470, 1240, 1050, $820 \mathrm{~cm}^{-1} ;{ }^{1} \mathrm{H} \mathrm{NMR}\left(\mathrm{CDCl}_{3}, 300 \mathrm{MHz}\right) \delta 7.32(\mathrm{~d}, J=8.4 \mathrm{~Hz}, 2 \mathrm{H}), 6.82(\mathrm{~d}, J=8.4 \mathrm{~Hz}, 2 \mathrm{H})$, $6.65(\mathrm{~s}, 1 \mathrm{H}), 6.55(\mathrm{~s}, 1 \mathrm{H}), 3.77(\mathrm{~s}, 6 \mathrm{H}), 3.35(\mathrm{~d}, J=1.5 \mathrm{~Hz}, 2 \mathrm{H}), 3.25(\mathrm{~d}, J=1.5 \mathrm{~Hz}, 2 \mathrm{H}) ;{ }^{13} \mathrm{C}$ $\mathrm{NMR}\left(\mathrm{CDCl}_{3}, 75 \mathrm{MHz}\right) \delta 170.7,158.3,149.7,149.3,130.8,129.9,129.1,121.3,121.2$, 120.8, 58.7, 53.2, 40.9, 39.7; HRMS (EI) calcd for $\mathrm{C}_{18} \mathrm{H}_{16} \mathrm{ClNO}_{4} \mathrm{~S}[\mathrm{M}]^{+}$377.0489, found 377.0565 .

3-Benzylimino-3,5-dihydro-7H-cyclopentan[c]thiopyran-6,6-dicarboxylic acid dimethyl ester (3ae, entry 5, reaction time: $12 \mathrm{~h}$ ).

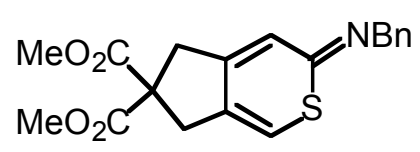

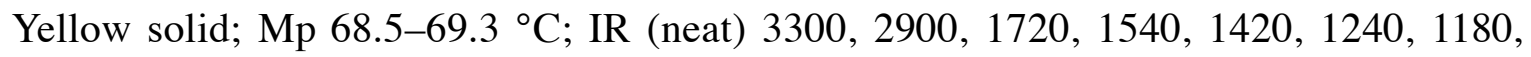
$1160,720 \mathrm{~cm}^{-1}$; ${ }^{1} \mathrm{H}$ NMR $\left(\mathrm{CDCl}_{3}, 300 \mathrm{MHz}\right) \delta 7.30-7.43(\mathrm{~m}, 4 \mathrm{H}), 7.20-7.28(\mathrm{~m}, 1 \mathrm{H}), 6.78(\mathrm{~s}$, $1 \mathrm{H}), 6.55(\mathrm{~s}, 1 \mathrm{H}), 4.25(\mathrm{~s}, 2 \mathrm{H}), 3.76(\mathrm{~s}, 6 \mathrm{H}), 3.32(\mathrm{~d}, J=1.8 \mathrm{~Hz}, 2 \mathrm{H}), 3.23(\mathrm{~d}, \mathrm{~J}=1.8 \mathrm{~Hz}, 2 \mathrm{H})$; ${ }^{13} \mathrm{C} \mathrm{NMR}\left(\mathrm{CDCl}_{3}, 75 \mathrm{MHz}\right) \delta 170.7,155.4,146.2,139.7,131.3,128.3,127.9,126.7,121.3$, 120.2, 58.8, 57.1, 53.2, 40.8, 40.0; HRMS (EI) calcd for $\mathrm{C}_{19} \mathrm{H}_{19} \mathrm{NO}_{4} \mathrm{~S}[\mathrm{M}]^{+}$357.1035, found 357.1055 .

3-Butylimino-3,5-dihydro-7H-cyclopenta[c]thiopyran-6,6-dicarboxylic acid dimethyl ester (3af, entry 5, reaction time: $20 \mathrm{~h}$ ).

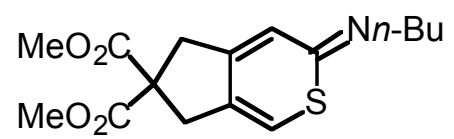

Yellow solid; Mp 76.5-77.0 ${ }^{\circ} \mathrm{C}$; IR (neat) 3200, 2900, 1720, 1540, 1420, 1240, 1180, 1150, $720 \mathrm{~cm}^{-1} ;{ }^{1} \mathrm{H} \mathrm{NMR}\left(\mathrm{CDCl}_{3}, 300 \mathrm{MHz}\right) \delta 6.74(\mathrm{~s}, 1 \mathrm{H}), 6.42(\mathrm{~s}, 1 \mathrm{H}), 3.75(\mathrm{~s}, 6 \mathrm{H}), 3.28(\mathrm{~d}, J=$ $1.2 \mathrm{~Hz}, 2 \mathrm{H}), 3.24(\mathrm{~d}, J=1.2 \mathrm{~Hz}, 2 \mathrm{H}), 3.00(\mathrm{t}, J=5.1 \mathrm{~Hz}, 2 \mathrm{H}), 1.74$ (quintet, $J=5.1 \mathrm{~Hz}, 2 \mathrm{H}$ ), $1.48-1.65$ (sextet, $J=5.1 \mathrm{~Hz}, 2 \mathrm{H}), 0.95(\mathrm{t}, J=5.1 \mathrm{~Hz}, 3 \mathrm{H}) ;{ }^{13} \mathrm{C} \mathrm{NMR}\left(\mathrm{CDCl}_{3}, 75 \mathrm{MHz}\right) \delta$ $170.8,154.5,145.7,131.9,121.0,120.4,58.7,53.2,53.1,40.7,40.0,32.7,20.9,14.0$; HRMS (EI) calcd for $\mathrm{C}_{16} \mathrm{H}_{21} \mathrm{NO}_{4} \mathrm{~S}[\mathrm{M}]^{+} 323.1191$, found 323.1167 .

3-Thioxo-3,5-dihydro-7H-cyclopenta[c]thiopyran-6,6-dicarboxylic acid dimethyl 
ester (3ag, entry 7, reaction time: $12 \mathrm{~h}){ }^{1}$

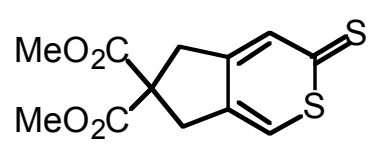

Red solid; Mp 127.2-127.8 ${ }^{\circ} \mathrm{C} ;{ }^{1} \mathrm{H} \mathrm{NMR}\left(\mathrm{CDCl}_{3}, 300 \mathrm{MHz}\right) \delta 7.44(\mathrm{~d}, J=1.2 \mathrm{~Hz}, 1 \mathrm{H})$, $7.40(\mathrm{~d}, J=1.2 \mathrm{~Hz}, 1 \mathrm{H}), 3.78(\mathrm{~s}, 6 \mathrm{H}), 3.45(\mathrm{~d}, J=1.5 \mathrm{~Hz}, 2 \mathrm{H}), 3.40(\mathrm{~d}, J=1.5 \mathrm{~Hz}, 2 \mathrm{H}) ;{ }^{13} \mathrm{C}$ $\mathrm{NMR}\left(\mathrm{CDCl}_{3}, 75 \mathrm{MHz}\right) \delta 204.4,170.3,150.8,136.2$, 135.1, 134.6, 58.7, 53.4, 41.0, 40.0.

1-(6-Acetyl-3-phenylimino-3,5,6,7-tetrahydrocyclopenta[c]thiopyran-6-yl)ethanone (3ba, entry 8, reaction time: $12 \mathrm{~h}){ }^{1}$

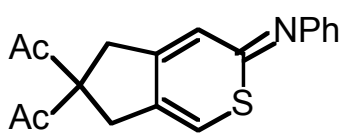

Red solid; Mp 121.5-122.0 ${ }^{\circ} \mathrm{C} ;{ }^{1} \mathrm{H}$ NMR $\left(\mathrm{CDCl}_{3}, 300 \mathrm{MHz}\right) \delta 7.30-7.40(\mathrm{~m}, 2 \mathrm{H})$, $7.05-7.15(\mathrm{~m}, 1 \mathrm{H}), 6.83-6.88(\mathrm{~m}, 2 \mathrm{H}), 6.63(\mathrm{~s}, 1 \mathrm{H}), 6.57(\mathrm{~s}, 1 \mathrm{H}), 3.27(\mathrm{~d}, J=1.5 \mathrm{~Hz}, 2 \mathrm{H})$, $3.16(\mathrm{~d}, J=1.5 \mathrm{~Hz}, 2 \mathrm{H}), 2.17(\mathrm{~s}, 6 \mathrm{H}) ;{ }^{13} \mathrm{C} \mathrm{NMR}\left(\mathrm{CDCl}_{3}, 75 \mathrm{MHz}\right) \delta 203.5,157.5,150.8$, $149.1,130.4,129.8,124.1,121.5,121.2,119.6,73.2,38.1,37.0,26.5$

1-(6-Acetyl-3-thioxo-3,5,6,7-tetrahydrocyclopenta[c]thiopyran-6-yl)ethanone (3bg, entry 9, reaction time: $12 \mathrm{~h})$.

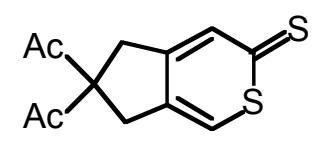

Red solid; Mp 111.4-112.0 ${ }^{\circ} \mathrm{C}$; IR (neat) 3200, 2950, 1500, 1400, 1350, 1170, $950 \mathrm{~cm}^{-1}$; ${ }^{1} \mathrm{H} \mathrm{NMR}\left(\mathrm{CDCl}_{3}, 300 \mathrm{MHz}\right) \delta 7.43(\mathrm{~s}, 1 \mathrm{H}), 7.41(\mathrm{~s}, 1 \mathrm{H}), 3.38(\mathrm{~d}, J=1.2 \mathrm{~Hz}, 2 \mathrm{H}), 3.34(\mathrm{~d}, J=$ $1.2 \mathrm{~Hz}, 2 \mathrm{H}), 2.19(\mathrm{~s}, 6 \mathrm{H}) ;{ }^{13} \mathrm{C} \mathrm{NMR}\left(\mathrm{CDCl}_{3}, 75 \mathrm{MHz}\right) \delta 204.2,202.6,150.6,136.0,135.3$, 134.9, 73.3, 38.1, 37.1, 26.4; HRMS (EI) calcd for $\mathrm{C}_{12} \mathrm{H}_{12} \mathrm{O}_{2} \mathrm{~S}_{2}$ [M-Ac] 209.0095, found 209.0175.

(6,6-Dimethoxymethyl-6,7-dihydro-5H-cyclopenta[c]thiopyran-3-ylidene)phenylamine (3ca, entry 10, reaction time: $12 \mathrm{~h}$ ).

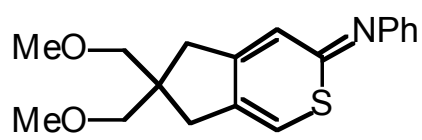


Yellow solid; Mp 94.5-95.5 ${ }^{\circ} \mathrm{C}$; IR (neat) 3300, 2800, 1520, 1090, 940, $860 \mathrm{~cm}^{-1} ;{ }^{1} \mathrm{H}$ NMR $\left(\mathrm{CDCl}_{3}, 300 \mathrm{MHz}\right) \delta$ 7.30-7.40 (m, 2H), 7.05-7.12 (m, 1H), 6.85-6.93 (m, 2H), 6.52-6.56 (m, 2H), 3.35 (s, 6H), $3.29(\mathrm{~s}, 4 \mathrm{H}), 2.67(\mathrm{~d}, J=1.5 \mathrm{~Hz}, 2 \mathrm{H}), 2.55$ (d, $J=1.5 \mathrm{~Hz}$, $2 \mathrm{H}) ;{ }^{13} \mathrm{C} \mathrm{NMR}\left(\mathrm{CDCl}_{3}, 75 \mathrm{MHz}\right) \delta 152.5,151.1,133.3,129.8,123.9,121.5,120.0,119.9$, 74.9, 59.3, 47.1, 39.3, 37.8; HRMS (EI) calcd for $\mathrm{C}_{18} \mathrm{H}_{21} \mathrm{NO}_{2} \mathrm{~S}[\mathrm{M}]^{+}$315.1293, found 315.1333.

\section{6,6-Dimethoxymethyl-6,7-dihydro-5H-cyclopenta[c]thiopyran-3-thione (3cg, entry} 11 , reaction time: $12 \mathrm{~h})$.

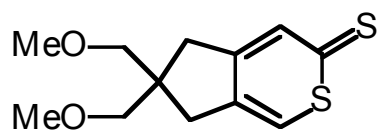

Yellow solid; Mp 80.4-80.9 ${ }^{\circ} \mathrm{C}$; IR (neat) 3300, 2800, 1580, 1400, 1150, $940 \mathrm{~cm}^{-1} ; \quad{ }^{1} \mathrm{H}$ NMR $\left(\mathrm{CDCl}_{3}, 300 \mathrm{MHz}\right) \delta 7.42-7.48(\mathrm{~m}, 1 \mathrm{H}), 7.32-7.38(\mathrm{~m}, 1 \mathrm{H}), 3.34(\mathrm{~s}, 6 \mathrm{H}), 3.30(\mathrm{~s}, 4 \mathrm{H})$, $2.77(\mathrm{~d}, J=1.2 \mathrm{~Hz}, 2 \mathrm{H}), 2.74(\mathrm{~d}, J=1.2 \mathrm{~Hz}, 2 \mathrm{H}) ;{ }^{13} \mathrm{C} \mathrm{NMR}\left(\mathrm{CDCl}_{3}, 75 \mathrm{MHz}\right) \delta 204.0,154.8$, 139.2, 135.8, 134.4, 74.9, 59.2, 47.3, 39.5, 38.1; HRMS (EI) calcd for $\mathrm{C}_{12} \mathrm{H}_{16} \mathrm{O}_{2} \mathrm{~S}_{2}[\mathrm{M}]^{+}$ 256.0592 , found 256.0522 .

\section{Rhodium-Catalyzed Enantioselective [2+2+2] Cycloaddition of 1,6-Diyne 1d with Isothiocyanate 2a (eq 3)}

(R)-(-)-6-Phenyl-3-phenylimino-3,5,6,7-tetrahydro-cyclopenta[c]thiopyran-6carboxylic acid methyl ester $[(R)-(-)-3 d a]$.<smiles>COC(=O)[C@@]1(c2ccccc2)Cc2csc(=[NH+]c3ccccc3)cc2C1</smiles>

Under an Ar atmosphere, $(R)$-BINAP $(18.7 \mathrm{mg}, 0.030 \mathrm{mmol})$ and $[\mathrm{Rh}(\mathrm{cod}) \mathrm{Cl}]_{2}(7.4 \mathrm{mg}$, $0.0150 \mathrm{mmol})$ were dissolved in $\mathrm{CH}_{2} \mathrm{Cl}_{2}(2.0 \mathrm{~mL})$ and the mixture was stirred at $\mathrm{rt}$ for $5 \mathrm{~min}$. $\mathrm{H}_{2}$ was introduced to the resulting solution in a Schlenk tube. After stirring at $\mathrm{rt}$ for $0.5 \mathrm{~h}$, the resulting mixture was concentrated to dryness. To the $\left(\mathrm{CH}_{2} \mathrm{Cl}\right)_{2}(4.0 \mathrm{~mL})$ solution of the residue was added a $\left(\mathrm{CH}_{2} \mathrm{Cl}\right)_{2}(1.0 \mathrm{~mL})$ solution of 2-phenyl-2-prop-2-ynylpent-4-ynoic acid methyl ester (1d, $67.9 \mathrm{mg}, 0.30 \mathrm{mmol})$ and phenyl isothiocyanate (2a, $44.6 \mathrm{mg}, 0.33 \mathrm{mmol})$ at $\mathrm{rt}$, and washed remaining substrates away by using $\left(\mathrm{CH}_{2} \mathrm{Cl}\right)_{2}(1.0 \mathrm{~mL})$. The mixture was stirred at $60{ }^{\circ} \mathrm{C}$ for $12 \mathrm{~h}$. The resulting mixture was concentrated and purified by silica gel 
column chromatography $\left(\mathrm{CH}_{2} \mathrm{Cl}_{2}\right)$, which furnished (R)-(-)-6-phenyl-3-phenylimino-3,5,6,7tetrahydrocyclopenta $[c]$ thiopyran-6-carboxylic acid methyl ester $[(\boldsymbol{R})-(-)-3 d a, 106.8 \mathrm{mg}$, $0.295 \mathrm{mmol}, 98 \%$ yield, $61 \%$ ee] as a yellow solid.

Mp 141.2-141.5 ${ }^{\circ} \mathrm{C} ;[\alpha]^{25}{ }_{\mathrm{D}}-6.40^{\circ}\left(\mathrm{CHCl}_{3}, c 2.405,61 \%\right.$ ee); IR (neat) 3200, 2900, 1700 , 1450, 1100, $680 \mathrm{~cm}^{-1} ;{ }^{1} \mathrm{H}$ NMR $\left(\mathrm{CDCl}_{3}, 300 \mathrm{MHz}\right) \delta 7.26-7.42(\mathrm{~m}, 7 \mathrm{H}), 7.10(\mathrm{t}, J=7.2 \mathrm{~Hz}$, $1 \mathrm{H}), 6.90(\mathrm{~d}, J=7.2 \mathrm{~Hz}, 2 \mathrm{H}), 6.66(\mathrm{~s}, 1 \mathrm{H}), 6.63(\mathrm{~s}, 1 \mathrm{H}), 3.74(\mathrm{~d}, J=16.5 \mathrm{~Hz}, 1 \mathrm{H}), 3.66(\mathrm{~d}, J$ $=16.5 \mathrm{~Hz}, 1 \mathrm{H}), 3.65(\mathrm{~s}, 3 \mathrm{H}), 3.14(\mathrm{dd}, J=16.5,1.2 \mathrm{~Hz}, 1 \mathrm{H}), 3.00(\mathrm{dd}, J=16.5,1.2 \mathrm{~Hz}, 1 \mathrm{H})$; ${ }^{13} \mathrm{C} \mathrm{NMR}\left(\mathrm{CDCl}_{3}, 75 \mathrm{MHz}\right) \delta 174.5,158.1,151.0,150.4,140.8,131.6,129.8,128.7,127.6$, 126.5, 124.0, 121.2, 120.6, 119.7, 57.1, 52.9, 43.0, 42.1; HRMS (EI) calcd for $\mathrm{C}_{22} \mathrm{H}_{19} \mathrm{NO}_{2} \mathrm{~S}$ $[\mathrm{M}]^{+}$361.1136, found 361.1094. CHIRALPAK AD, hexane:2-PrOH = 85:15, $1.0 \mathrm{~mL} / \mathrm{min}$, retention times: $12.1 \mathrm{~min}$ (major isomer) and $15.1 \mathrm{~min}$ (mimor isomer).

\section{References}

(1) Yamamoto, Y.; Kinpara, K.; Saigoku, T.; Takagishi, H.; Okuda, S.; Nishiyama, H.; Itoh, K. J. Am. Chem. Soc. 2005, 127, 605-613.

(2) Bhar, S.; Chaudhuri, S. K.; Sahu, S. G.; Panja, C. Tetrahedron 2001, 57, 9011-9016.

(3) Cadran, N.; Cariou, K.; Herve, G.; Aubert, C.; Fensterbank, L.; Malacria, M.; MarcoContelles, J. J. Am. Chem. Soc. 2004, 126, 3408-3409.

(4) Madine, J. W.; Wang, X.; Widenhoefer, R. A. Org. Lett. 2001, 3, 385-388. 

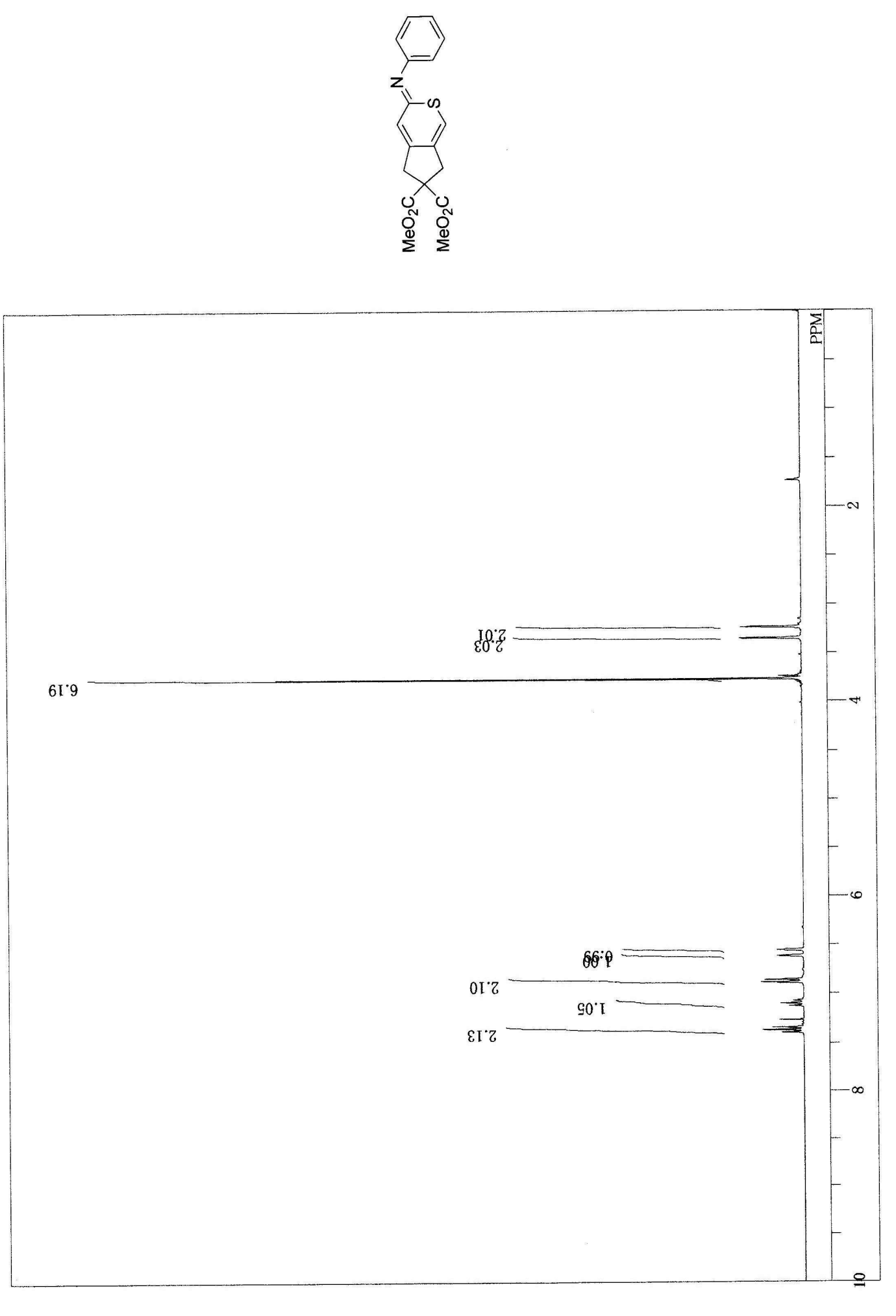

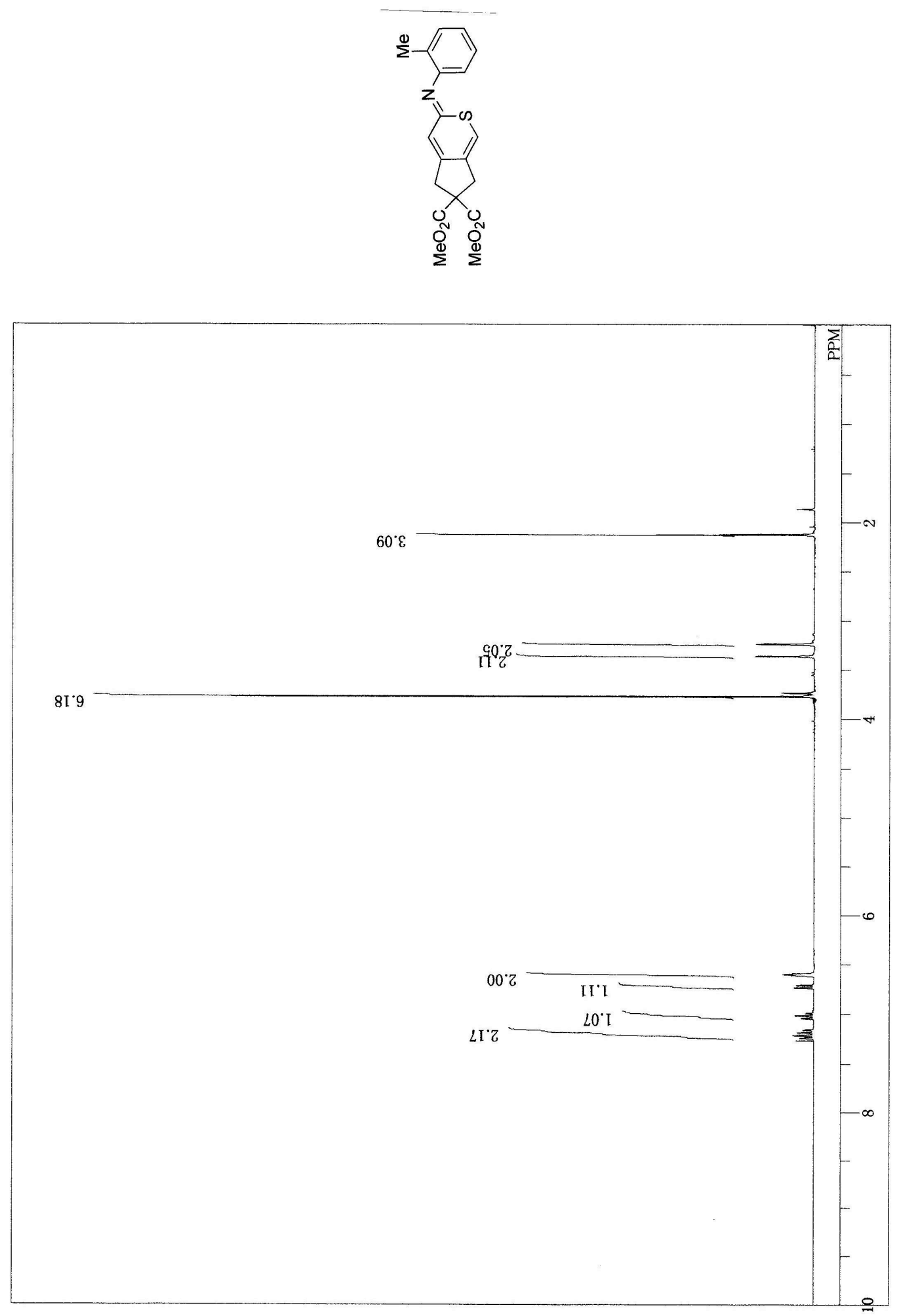

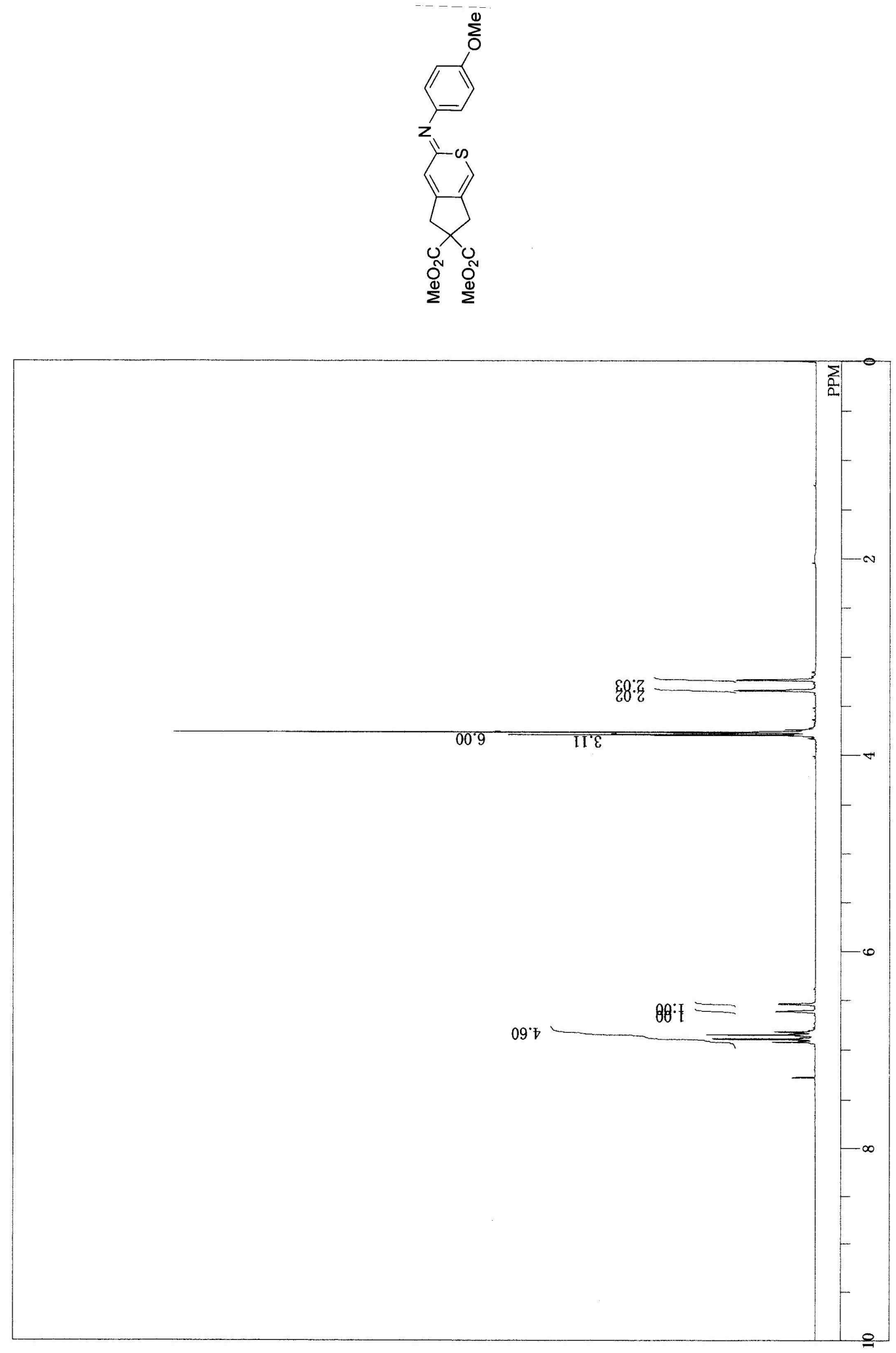

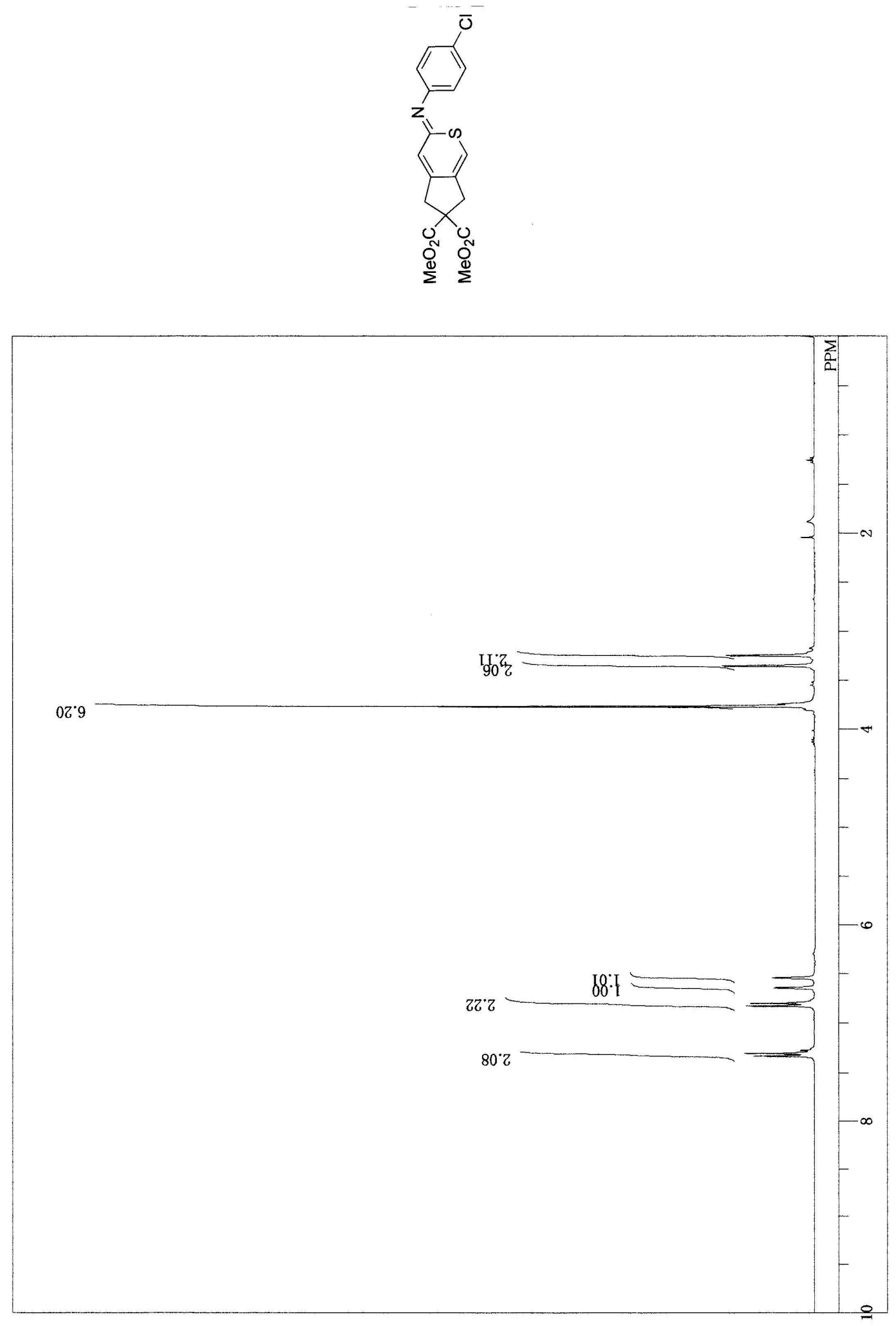

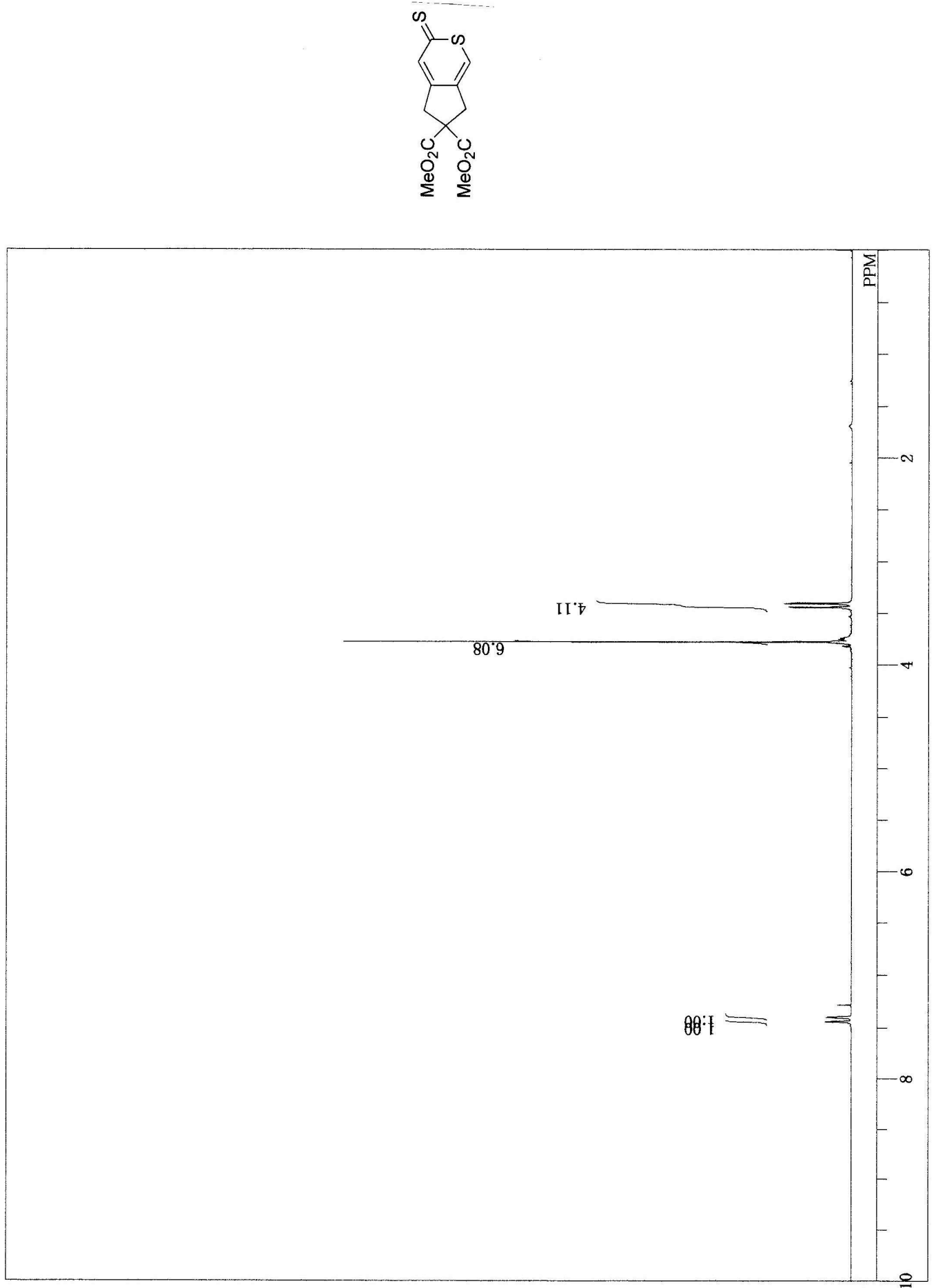

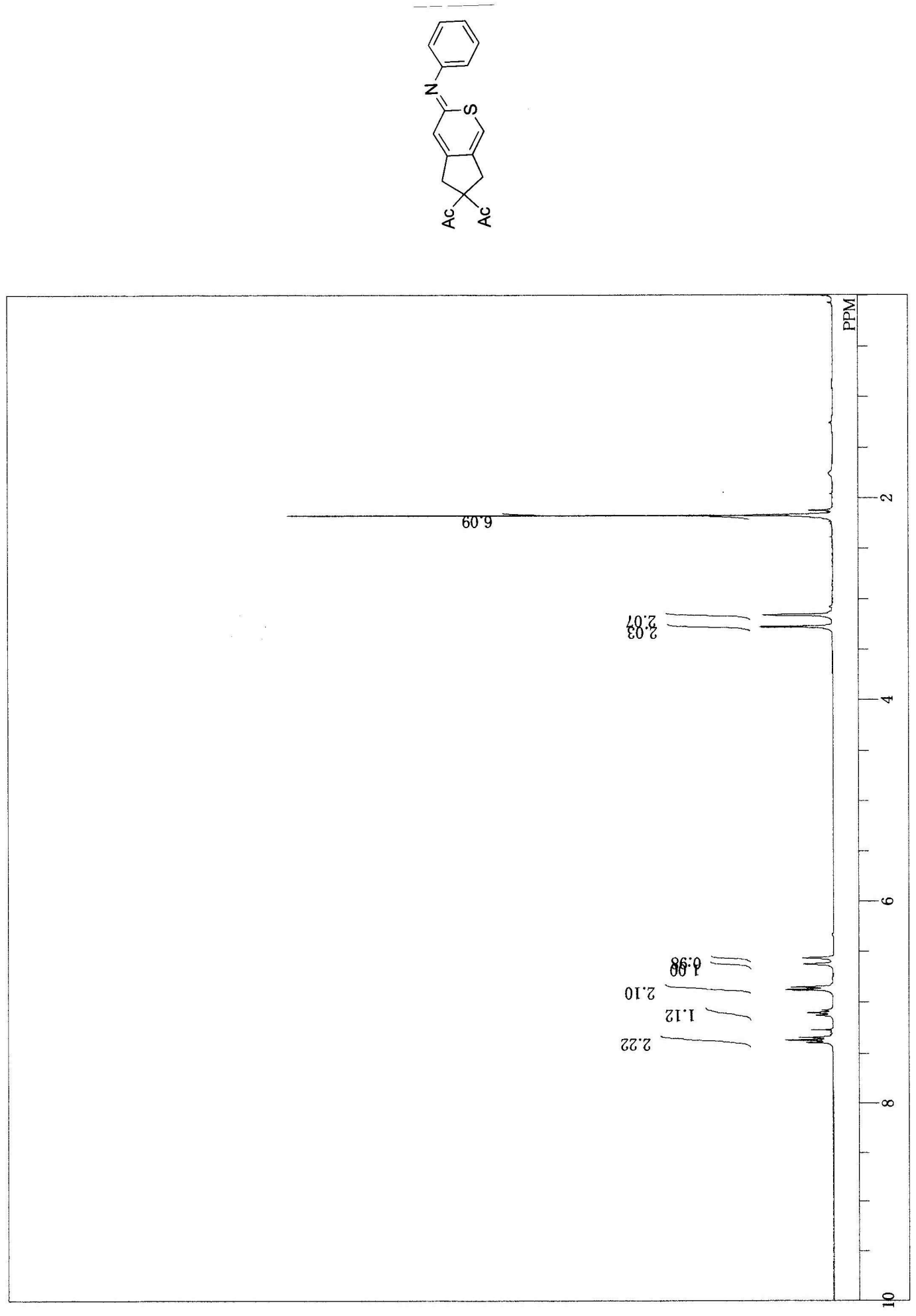

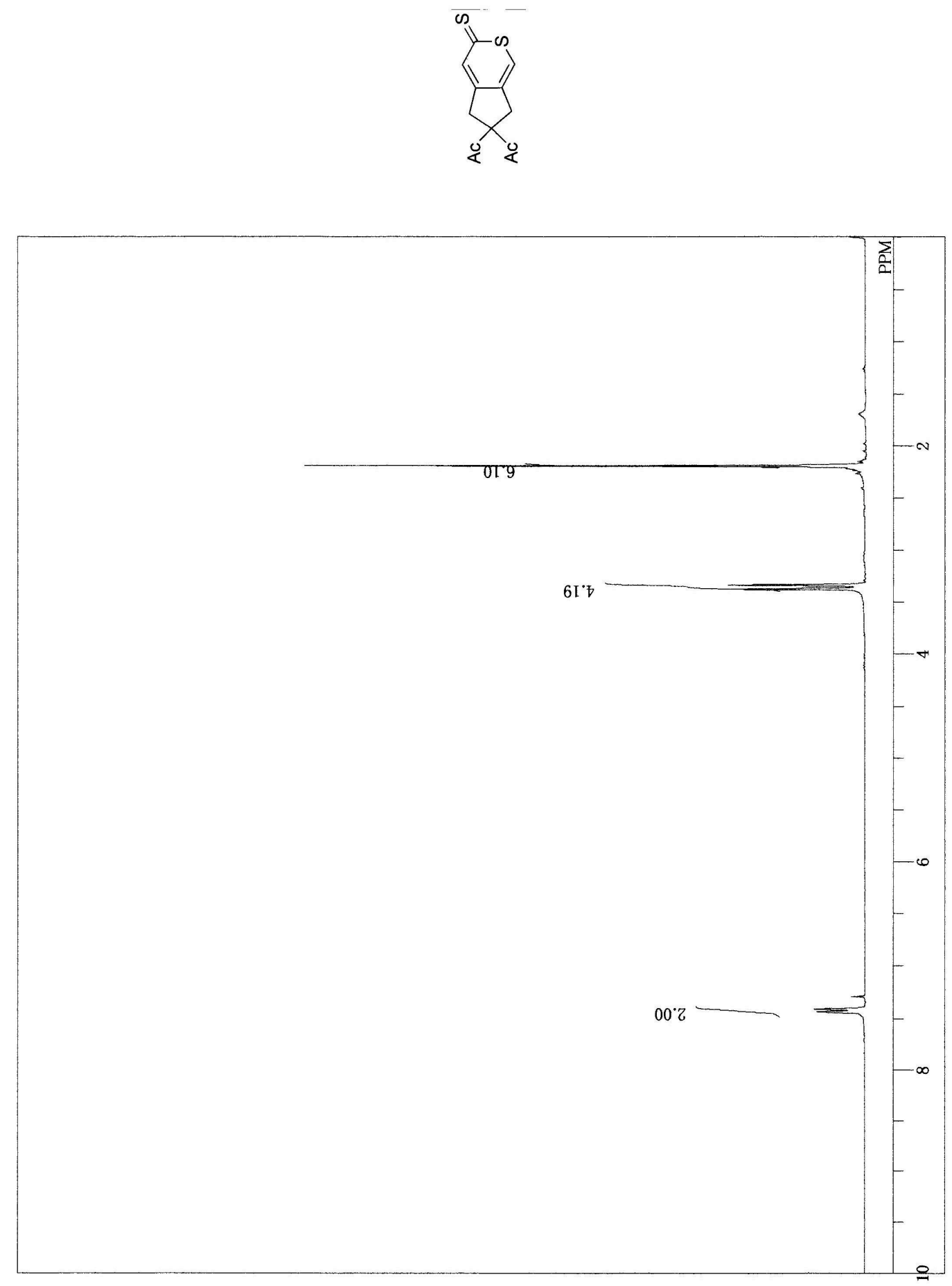

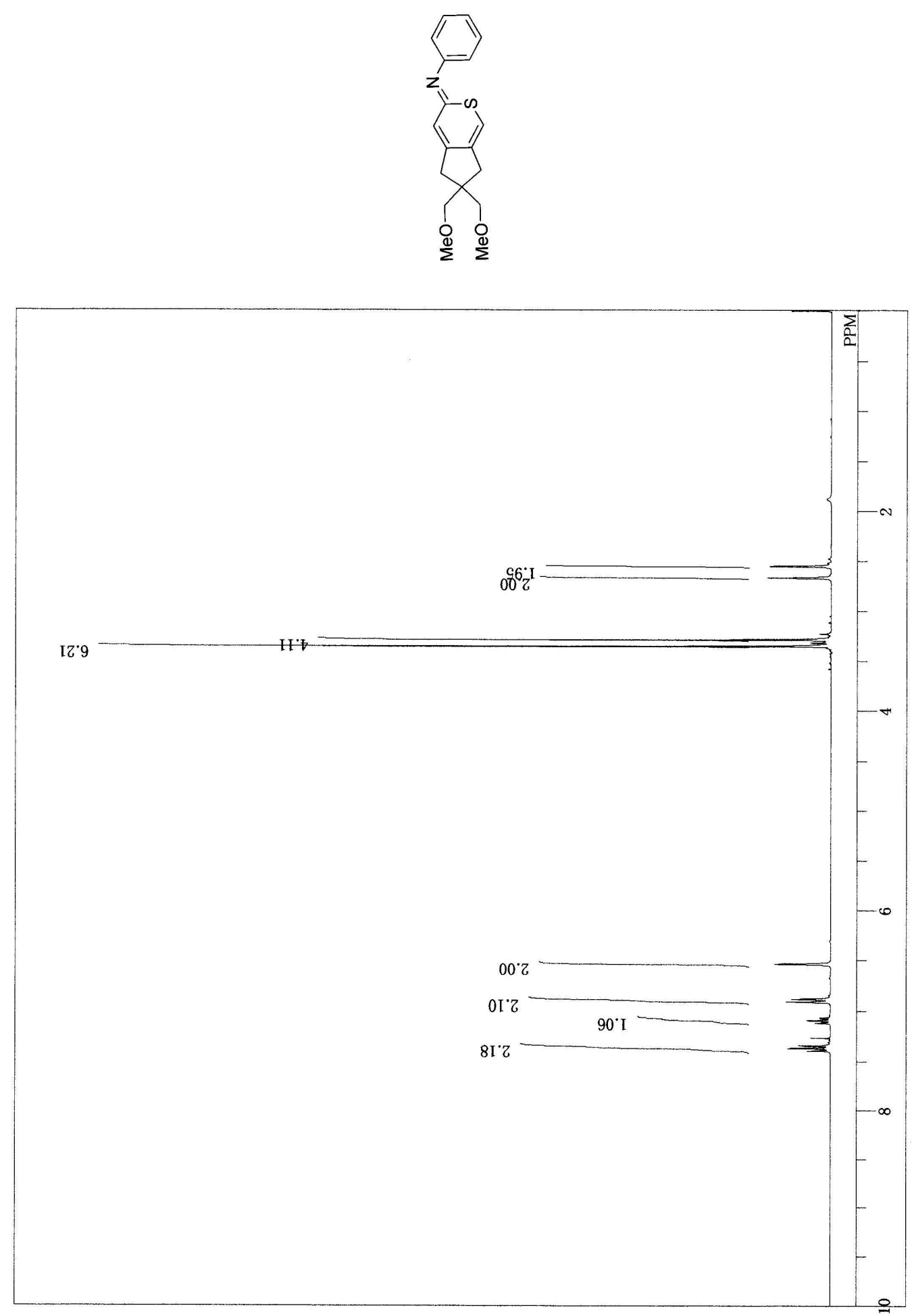

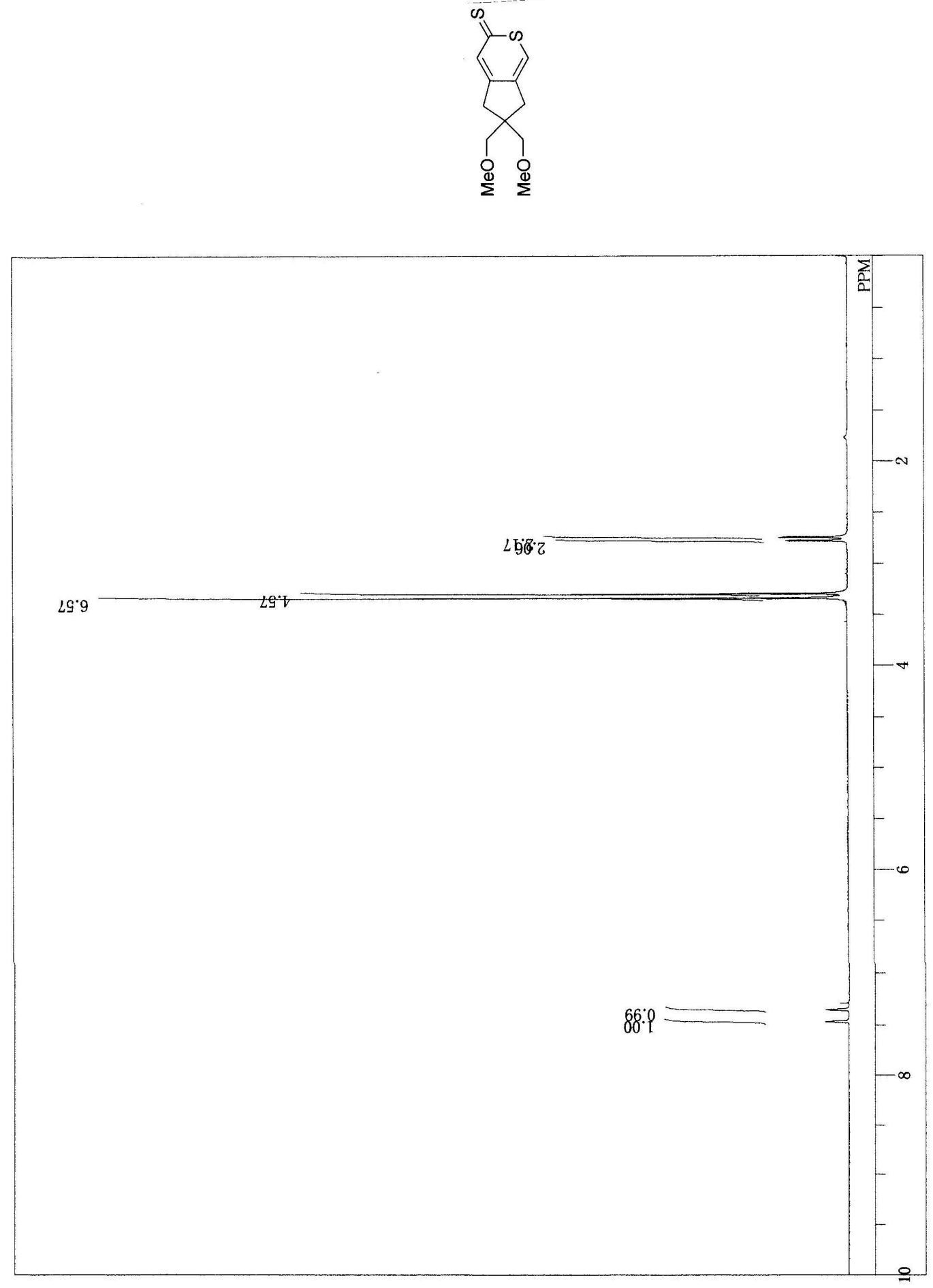

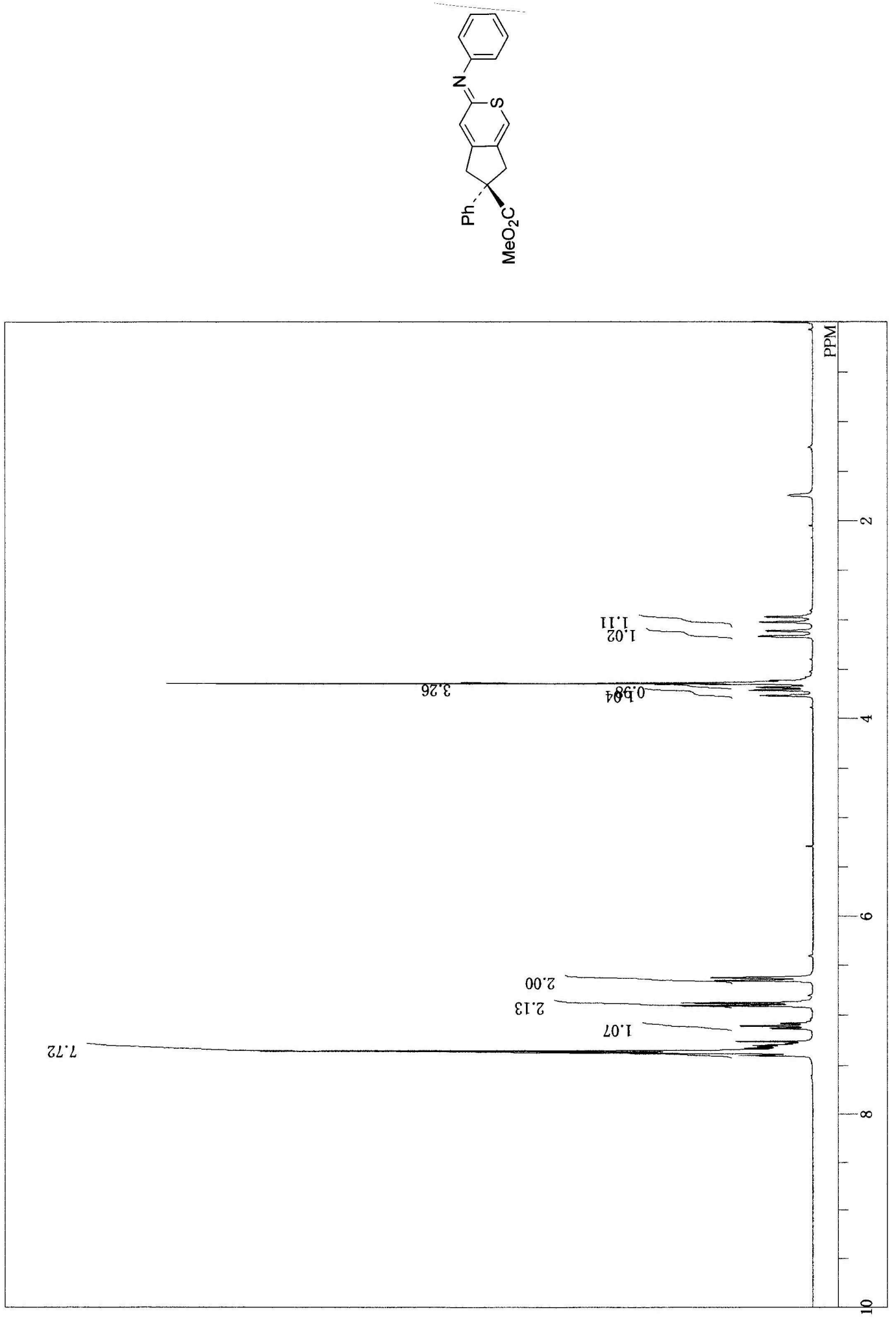